\title{
Adapting the Failure Modes Effect Analysis (FMEA) for Early Detection of Human Factors Concerns
}

Judy Village

Ryerson University

\section{Tim Annett}

Research in Motion Ltd

\section{Edward Lin}

Research in Motion Ltd

\section{Michael Greig}

Ryerson University

\section{Patrick W. Neumann}

Ryerson University

digital.library.ryerson.ca/object/282

\section{Please Cite:}

Village, J., Annett, T., Lin, E., Greig, M., \& Neumann, P. W. (2011). Adapting the failure modes effect analysis (FMEA) for detection of human factors concerns. Proceedings of the 42nd Annual Conference of the Association of Canadian Ergonomists, 17-20.
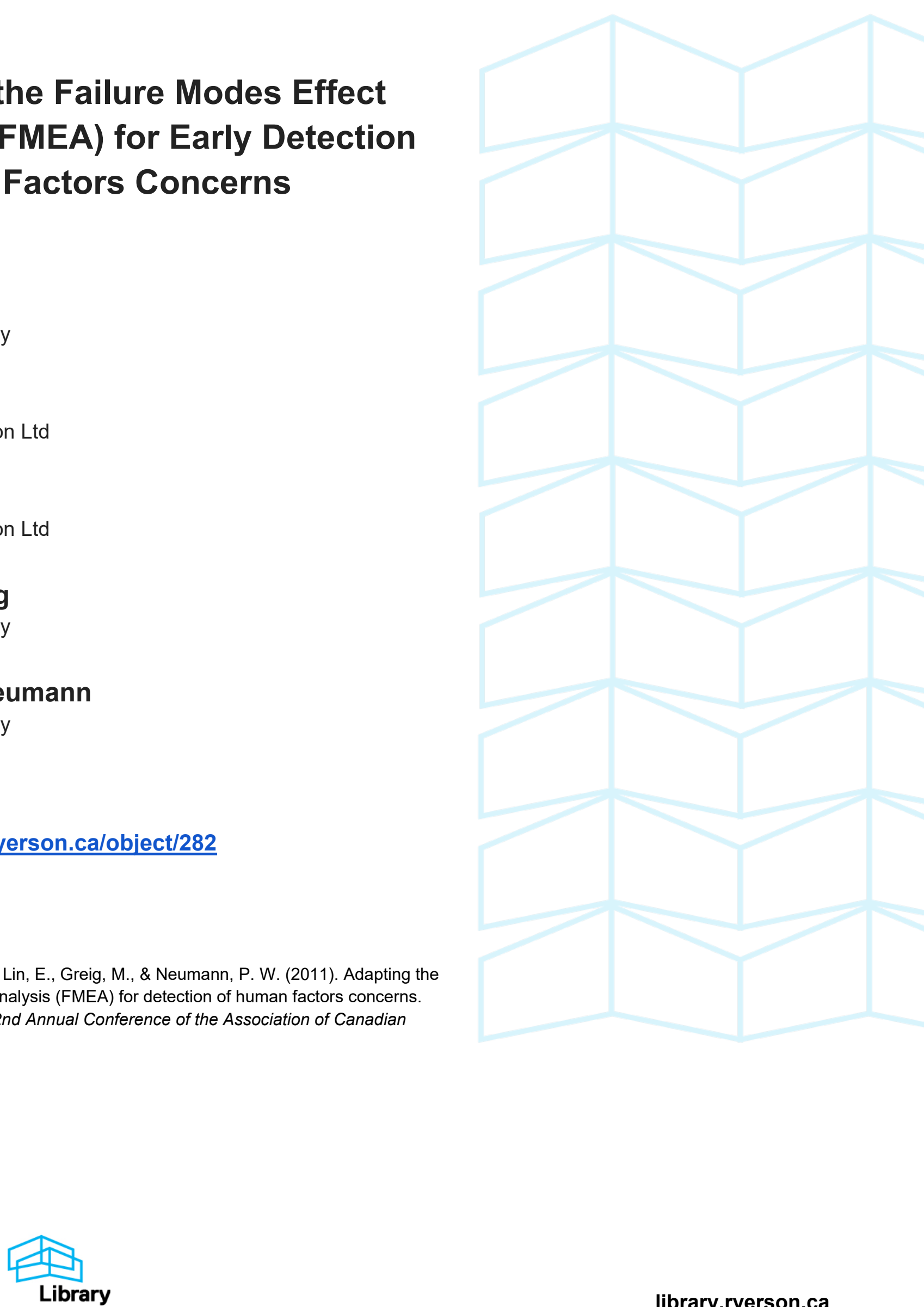


\title{
ADAPTING THE FAILURE MODES EFFECT ANALYSIS (FMEA) FOR EARLY DETECTION OF HUMAN FACTORS CONCERNS
}

\author{
Judy Village \\ Ryerson University, Human Factors Engineering Lab, Department of Mechanical and \\ Industrial Engineering, 350 Victoria St., Toronto, Ontario M5B 2K3 \\ Tim Annett, Edward Lin \\ Research in Motion Ltd., Ergonomics Group, Waterloo, Ontario \\ Michael Greig, Patrick W. Neumann \\ Ryerson University, Toronto, Ontario
}

\begin{abstract}
As one of many initiatives underway in a collaborative action research project with a large manufacturer, this paper presents the development of a "human factors" failure modes effect analysis (HF-FMEA). FMEA is an engineering reliability tool that helps define, identify, prioritize and eliminate known or potential failures of a system, design or manufacturing assembly process, generally to optimize quality or systems safety for consumers. The goal of the HF-FMEA is to detect and minimize risk of injury for the operator who will assemble products, prior to design of an assembly line. Scoring procedures for "severity", "occurrence" and "detection" from a HF perspective are presented with examples. Embedding the HFFMEA into software templates, and structuring a process for support and integration helps ensure its continued use. The process may be useful for other organizations with handintensive assemblies to optimize worker health together with assembly quality.
\end{abstract}

Keywords: FMEA, human factors, systems design

\section{DÉTECTION DES PROBLÈMES VISANT LES FACTEURS HUMAINS}

Comme beaucoup d'autres initiatives entreprises dans le cadre d'un projet de rechercheaction réalisé en collaboration avec un grand fabricant, ce document présente la conception d'une analyse des modes de défaillance et de leurs effets par rapport aux facteurs humains (AMDE-FH). Une AMDE est un outil sur les techniques de fiabilité qui aide à définir, déterminer, hiérarchiser et éliminer les défaillances possibles ou connues d'un système, d'une conception ou d'un processus de fabrication à la chaîne, afin d'optimiser la qualité ou la sécurité des systèmes pour les utilisateurs. L'objectif de l'AMDE-FH vise à détecter et à réduire les risques de blessure pour le travailleur à la chaîne, avant la conception d'une chaîne de montage. Les critères d'évaluation " gravité ", " occurrence » et " détection », en fonction des facteurs humains, seront présentés avec des exemples. Incorporer l'AMDE-FH dans un modèle de logiciel et structurer un processus de soutien et d'intégration permet d'en assurer l'usage continu. Le processus peut servir à d'autres organisations équipées de chaînes de montage pour optimiser la santé du travailleur à la chaîne ainsi que la qualité de son travail.

Mots clés : AMDE, facteurs humains, conception de systèmes 


\section{$1.0 \quad$ INTRODUCTION AND OBJECTIVES}

Ryerson University's Human Factors Engineering Laboratory is working with a large manufacturer to integrate human factors (HF) into the production system design process with a combined goal of reduced occupational health concerns for workers and improved quality of production. Various initiatives are underway, one of which involves development and implementation of a "human factors" (HF) failure modes and effects analysis (HF-FMEA). The FMEA is a proactive process to predict quality problems that may arise during design or assembly of products. This paper presents the development of a HF-FMEA to predict and control assembly problems from the perspective of the operator before the assembly line is designed.

\subsection{Background of FMEAs}

FMEAs were first used in the aerospace industry in the 1960s, and were later brought into mainstream by the automotive industry (Ginn et al., 1998). FMEAs are routinely carried out by engineering and quality teams to minimize quality problems prior to design of products, processes or systems. The FMEA is a requirement of the QS-9000 quality system (1994) for automotive companies (Arunachalam et al., 2002). The healthcare sector also requires an FMEA risk assessment yearly focused on patient safety, to maintain accreditation (Joint Commission on Accreditation of Health Care Organizations, 2004) (Habraken et al., 2009).

The basic process for an FMEA involves assembling a team with the engineering expertise to determine all potential failure modes with a product, process or system. Failure modes may be the result of a faulty component, assembly process, or operator error. For each potential failure, effects of the failure on the consumer are listed and rated on a 1-10 scale based on the severity of the effect, occurrence or likelihood of the failure happening, and ability to detect the failure before it happens. The three scores are multiplied together yielding a risk priority number (RPN) that ranges from 1 to 1000. Cut-offs (generally at 100), help prioritize which failures require actions to reduce or control the failure effects.

Human factors FMEAs have been reported for systems failures (such as the shuttle dome heating problem) (Rong and Zhao, 2008). They have also been used to detect human error termed Error Modes Effects Analysis (EMEA) (Lehto, 2000). Only two publications were found that discussed using FMEAs from the perspective of operator health as the failure, rather than product, process or systems failures (Barsky and Dutta, 1997; Munck-Ulfsfalt, 2004). The "cost assessment for ergonomics risk" (CAFER) is a theoretical model that determines the cost of injury expense attributable directly to a product or process designed in a manner that risk of injury is present (Barsky and Dutta, 1997). Munsck-Ulfsalt (2004) discusses a risk assessment FMEA based on a series of checklists for integrating ergonomics into the whole chain from design to manufacturing, with requirement specifications on the product, process, organization, logistics and workplace. While they termed their approach an FMEA, there was no attempt to mimic the severity, occurrence and detection scales of typical FMEAs.

\subsection{FMEA Process in Participating Manufacturing Company}

In our partner company, design FMEAs are first performed to ensure the product meets market needs. These then inform assembly process FMEAs, which are performed at three stages of a products' life: prototype, pre-launch, and production. The FMEA team is assembled with engineers from process design, production, testing, quality, manufacturing, and supplier quality. 
Currently in the company, Ergonomists perform assessments and recommend solutions when an incident report is completed by an injured worker. The goal of the HF-FMEA is to proactively detect and prevent these assembly problems, before designing the assembly process, resulting in benefits for operator well-being and product quality.

\section{$2.0 \quad$ METHODS}

The project uses an action research methodology in which organization participants and researchers jointly pursue action (or change) and research (or understanding) at the same time by using collaborative spirals of planning, acting, observing, reflecting and re-planning (Meyer, 2000). The goal is for HF to become seamless and embedded in existing processes. To develop the HF-FMEA, 4 Ergonomists (2 from the company, and 2 from Ryerson) attended and observed FMEA meetings to understand how quality FMEAs proceed. Notes of human factors related issues were compared after the meetings.

A review of the scientific literature was conducted for HF-specific methods. The 4 ergonomists worked collaboratively to determine possible options based on the scientific literature for definitions and ratings of "severity" (degree of risk), "occurrence" (probability of risk) and "detection" (likelihood a risk control method will detect and prevent an occurrence). Three options were agreed to for pilot testing. A HF-FMEA worksheet was drafted consistent with the current quality approach. To pilot test the various scoring options, a previously completed quality FMEA was used in conjunction with observations and discussions with workers on the resulting assembly line currently operating. Using the identified failure modes on the quality FMEA, each task was observed and scored with the three options. The ergonomists then reviewed scores and problems, and agreed to one final scoring option.

A further pilot test was conducted to ensure the scoring and resulting risk priority number was reliable between scorers, and had face validity with respect to other ergonomic risk assessment procedures. Using videotapes from the above-mentioned assembly line, each ergonomist scored the failure modes where human factors related assembly tasks were identified. Scores were compared and discussed. Tasks were also evaluated using rapid upper body assessment (RULA) and results were compared with the cut-point RPN of 100 which should indicate the need for corrective action.

Methods of integrating the HF-FMEA into current design processes at the company will be discussed in the next section, as this continues to be a work in progress.

\subsection{RESULTS OF HF-FMEA DEVELOPMENT PROCESS}

From observation of quality FMEA meetings, Ergonomists noted many tasks identified as a quality failure mode that also appeared to pose potential difficulties and risk for operators. Examples include misaligned screws, improper alignment of connectors, or excessive force to connectors. Often the risk control for these failure modes was to improve the training or instructions for operators, or to build a jig to ensure screws are aligned correctly.

Ergonomists reviewed the scoring in place for quality FMEAs, and also reviewed scoring systems and modifications in the literature for guidance. Severity is generally performed subjectively by the FMEA team based on their experience with previous assembly processes. For the HF-FMEA, we defined severity as the risk of injury to the operator with " 10 " representing a high risk that the task will cause injury. In an effort to provide a method that is semi-quantitative for rating the severity risk, we modified the cube model (Kadefors, 1994). 
The cube model is a practical assessment that involves rating of force $(F)$, postural strain $(P)$, and repetition (R) on a three point scale of low (1), medium (2) or high (3) (Table 1), and is most useful for hand-intensive tasks (Laring et al, 2002). Multiplication of the three values results in a score from 1-27, with scores above 10 considered "unacceptable". Since the tasks in assembly are generally low force, but can contain considerable static work and other risk factors (eg. contact stress, hand-arm vibration and high visual demands), we adjusted the score (by adding 1) if significant other risks factors (RF) were apparent. Using the suggested cut-offs in the cube model, the score was then adjusted to a 1-10 scale consistent with the FMEA (Table 2). Since the scoring is applied prior to design of an assembly line, actual repetition rates may not be known. Therefore, practical use of the score involves some approximation or anticipation based on previous experience with other assembly lines.

Table 1. Modified Cube Ratings for Force (F), Posture (P), and Repetition (R)

\begin{tabular}{|c|c|c|c|}
\hline Score & Force (F) & Posture (P) & Repetition (R) \\
\hline 1 (low) & $\begin{array}{l}<1 \mathrm{~kg} \text { or } 10 \% \\
\text { MVC }\end{array}$ & Optimal posture $\left(<15^{\circ}\right)$ & $\begin{array}{l}<0.1 \mathrm{c} / \text { min shoulder } \\
<1 / \text { min hand } \\
<20 / \text { min fingers }\end{array}$ \\
\hline 2 (med) & $10-25 \% \mathrm{MVC}$ & $\begin{array}{l}\text { Near optimal posture } \\
\text { Shoulder } 15-45^{\circ}\end{array}$ & $\begin{array}{l}0.1-2.5 \mathrm{c} / \mathrm{min} \text { shoulder } \\
1-10 / \mathrm{min} \text { hand } \\
20-200 / \mathrm{min} \text { fingers }\end{array}$ \\
\hline 3 (high) & $\begin{array}{l}>2.3 \mathrm{~kg} \text { single } \\
\text { hand, } 25 \% \mathrm{MVC}\end{array}$ & $\begin{array}{l}\text { Hand above shoulder height } \\
\text { Arm extended } \\
\text { Shoulder abd }>45^{\circ}\end{array}$ & $\begin{array}{l}>2.5 / \text { min shoulder } \\
>10 / \text { min hand } \\
>200 / \text { min fingers }\end{array}$ \\
\hline
\end{tabular}

Table 2. The product of force $(F)$, posture $(P)$ and repetition $(R)$ score is added to other risk factors (RF) for a total score, which is converted to a 1-10 severity (S) score

\begin{tabular}{|l|l|l|l|l|}
\hline $\mathbf{F}^{*} \mathbf{P}^{*} \mathbf{R}$ & $\begin{array}{l}\mathbf{+ 1} \text { for other risk } \\
\text { factors (RF) }\end{array}$ & $\begin{array}{l}\text { Total } \\
\text { Score }\end{array}$ & $\begin{array}{l}\text { Severity } \\
\text { conversion }\end{array}$ & Severity Risk (S) \\
\hline & & $1-4$ & $1-3$ & Low level risk factors \\
\hline & & $5-7$ & $4-5$ & Low risk with 1 medium risk factor \\
\hline & & 8 & $6-7$ & Medium risk factors \\
\hline & & 9 & $8-9$ & Medium with 1 high risk factor \\
\hline & & $\geq 10$ & 10 & High level risk factors \\
\hline
\end{tabular}

Occurrence ratings in quality are generally based on data indicating the probability of each failure mode, with "10" representing very high persistent failures (eg. >100 per thousand pieces). For the HF-FMEA, we considered defining the probability based on the percentage of the cycle time that risk factors are present. We also considered defining likelihood in terms of the number of hours per shift that the worker is exposed to the task. Both of these methods proved difficult in practice since this information may be unknown prior to design of the assembly line. In the CAFER model, consideration was given as to the part of body likely to be injured, average cost of injury to that body part, the number of workers exposed to the task, and the probability of injury (based on previous records) (Barsky and Dutta, 1997). In other studies, probability is determined subjectively based on the experience of the group and previous history (Chiozza and Ponzetti, 2009). Our group settled on a qualitative rating of occurrence (Table 3 ) in which the team considers the part of body to which the injury may occur (eg. wrist), the type of injury that might occur (eg. tendonitis), the extent to which research supports a relationship between the risk factors and type of injury (eg. strong evidence), the severity (scored based on risk factors), frequency and duration of exposure, and experience of previous history of concerns or injuries with the task or a similar task. 
Detection generally refers to the ability of the failure mode to be detected or controlled automatically. In the HF-FMEA, detection is defined as the ability of the control, if necessary because of high risk, to reduce or eliminate risk factors (Table 3 ). This use of detection is designed to encourage engineering risk control to reduce or eliminate risk factors, thereby reducing the overall RPN score. Consistent with quality FMEAs, the risk priority number (from $1-1,000)$ is the product of severity $(S)$, occurrence $(O)$ and detection $(D)$, with a score $\geq 100$ indicating need for risk control. Table 4 shows an example using two sample assembly tasks.

Table 3. Verbal Anchors for Ratings of Occurrence (O) and Detection (D)

\begin{tabular}{|l|l|l|l|}
\hline $\mathbf{O}$ & Description & D & Description \\
\hline $1-2$ & Very unlikely to cause injury & $1-2$ & $\begin{array}{l}\text { Risk factors either not present or } \\
\text { eliminated with controls }\end{array}$ \\
\hline $3-4$ & Low probability of injury & $3-4$ & $\begin{array}{l}\text { Risk factors are low or if present, } \\
\text { controlled with engineering controls }\end{array}$ \\
\hline $5-6$ & May cause injury to some workers & $5-6$ & $\begin{array}{l}\text { Risk factors are medium, or with some } \\
\text { control but some risk remains }\end{array}$ \\
\hline $7-8$ & Likely to cause injury to some & $7-8$ & $\begin{array}{l}\text { Risk factors present, controls in place are } \\
\text { ineffective }\end{array}$ \\
\hline $9-10$ & High likelihood to cause injury & $9-10$ & $\begin{array}{l}\text { Risk factors insufficiently controlled, high } \\
\text { need for risk control }\end{array}$ \\
\hline
\end{tabular}

Table 4. Sample assembly tasks with ratings of force $(F)$, posture $(P)$, repetition $(R)$ and other risk factors (RF), and associated severity (S), occurrence (O) and detection (D) scores to determine the risk priority number (RPN)

\begin{tabular}{|l|c|c|c|c|c|c|c|c|r|}
\hline Assembly Task & $\mathbf{F}$ & $\mathbf{P}$ & $\mathbf{R}$ & $\mathbf{R F}$ & $\mathbf{( F ^ { * } \mathbf { P } ^ { * } \mathbf { R } ) + \mathbf { R F }}$ & $\mathbf{S}$ & $\mathbf{O}$ & $\mathbf{D}$ & $\mathbf{R P N}$ \\
\hline $\begin{array}{l}\text { Pick up A, insert connectors (2 } \\
\text { motions/2-14 sec) }\end{array}$ & 1 & 1 & 3 & 1 & 4 & 3 & 4 & 4 & 48 \\
\hline $\begin{array}{l}\text { Pick up C and D, insert at 30 deg angle, } \\
\text { get C, attach and snap (5 motions/20s) }\end{array}$ & 1 & 2 & 3 & 1 & 7 & 5 & 4 & 5 & 100 \\
\hline
\end{tabular}

In the first task in Table 4, the force and posture were low risk and repetition was high. There was an additional risk from static loading to yield a severity score of 3 . Occurrence and detection were rated as 4 indicating that the probability of injury was fairly low and there were some controls in place. The overall RPN (48) does not indicate the need for risk control. The second task differs as there is a moderate level of force and additional visual demands yielding a severity rating of 5 . The score of 5 for detection indicates that risk remains resulting in an RPN of 100, necessitating risk control to reduce the RPN.

\subsection{DISCUSSION OF HF-FMEA IMPLEMENTATION}

Senior directors of the company provided strong support for the HF-FMEA initiative and the timing for development fortunately coincided with an upgrade of their FMEA software. The HF-FMEA will have a separate template within the software with drop-down windows for efficient scoring of each factor and automatic calculation of RPNs. Having the HF-FMEA built into the companies' software further anchors it within the organization since the software automatically produces a work order with action items and persons responsible.

To further ingrain the initiative into the engineering culture and ensure adequate status, support, and buy-in, it was drafted into a project "charter". This process defines a sponsor 
(Senior Director), an overseeing committee, action items, timelines, and deliverables. Three phases are anticipated for the HF-FMEA. In the first phase, Ergonomists will attend FMEA meetings to increase awareness and gradually introduce engineers to the HF scoring. Ergonomists will also document examples from these meetings where risk control has reduced the RPN, and thus the risk of injury to workers. This helps engineers see the impact and provides a means of benchmarking the current situation to compare with the future scores and solutions. Phase two will include training for engineers in the HF-FMEA scoring, and reduced direct participation by ergonomists in FMEAs to help phase the HF-FMEA into regular processes. Ergonomists will continue to document HF RPN scores pre-and post risk control. In the third phase, Ergonomists will monitor the HF-FMEA from a distance, and continue more limited direct support to facilitate integration within engineering.

A challenge Ergonomists encountered in developing the HF-FMEA was the cross-disciplinary nature of the FMEA team, making it difficult to identify essential players in the organization. Numerous departments with different managers are involved, including new product engineering, continuous improvement, quality, manufacturing, and human factors, the latter being a separate department outside of engineering and manufacturing.

\subsection{CONCLUSIONS}

A HF-FMEA was developed for light assembly work that scores risk of injury to the operator prior to designing the assembly line. This has the potential to proactively minimize injury hazards before they reach the shop floor. Designing a HF-FMEA template that has similar scoring and cut-offs to quality FMEAs, and embedding it in newly developed software should help facilitate ongoing buy-in and use by engineers. The process of developing the HF-FMEA may be useful for other organizations working to integrate HF into design processes.

\section{ACKNOWLEDGEMENTS}

This work has been made possible by funding from the Natural Science and Engineering Research Council of Canada (NSERC) and by the Workplace Safety and Insurance Board of Ontario (WSIB).

\section{REFERENCES}

Arunachalam, S, McAndrew, I., O'Sullivan, J.M., Gunasekaran, A. (2002). Implications of QS9000 in the implementation of a new technology: a case study. Int. J. Manufacturing Technology and Management, 4(3/4), 322-333.

Barsky, I, Dutta, S.P. (1997). Cost assessment for ergonomic risk (CAFER). International Journal of Industrial Ergonomics, 20, 307-315.

Chiozzas, ML, Ponzetti, C. (2009). FMEA: A model for reducing medical errors. Clinica Chimica Acta, 404, 75-78.

Ginn, DM, Jones, D.V., Rahnejat, H., Zairi, M. (1998). The 'QFD-FMEA interface“. European Journal of Innovation Management, 1(1), 7-20.

Habraken, MMP, Van der Schaaf, T.W., Leistikow, L.P., Reijnders-Thijssen, P.M.J. (2009). Prospective risk analysis of health care processes: A systematic evaluation on the use of HFMEA in Dutch health care. Ergonomics, 59(7), 809-819.

Kadefors, R. (1994). An ergonomic model for workplace assessment. Proc. of IEA'94, Vol. 5. International Ergonomics Association, Toronto, Canada, 210-212.

Laring, J., Forsman, M., Kadefors, R., Ortengren, R. (2002). MTM-based ergonomic workload analysis. Int. J. Ind. Ergo, 30, 135-148. 
Lehto, MR. (2000). Determining warning label content and format using FMEA. Proceedings of the IEA 2000/HFES; San Diego, USA, 774-778.

Meyer, J. (2000). Qualitative research in health care. Using qualitative methods in health related action research. British Medical Journal, 320, 178-181.

Munsck-Ulfsalt, U. (2004). Requirement specification for load-ergonomic risk assessment with FMEA-Checklists-A Model for risk assessment from design to production. Nordic Ergonomics Society $56^{\text {th }}$ Annual Conference, Keldling, Denmark, 149-152.

Rong, M, Zhao, T., Yu, Y. (2008). Advanced Human Factors Process Failure Modes and Effects Analysis. Annual Reliability and Maintainability Symposium, Las Vegas, NV, USA, 365-370. 